\title{
Visceral Data - Observations of Point Clouds
}

\author{
LESLIE FOREHAND
}

lowa State University

In the essay 'Clouds', Marcel Beyer describes an enduring year studying and elucidating the scholarly production of clouds. He proceeds to describe nephology, or the study of clouds, as a vertigo-inducing process because 'the object under observation - be it an individual cloud photograph, be it scientific cloud observation as a whole - ceaselessly asks back: How do you observe?.' This paper extends the same process of observation into clouds of data, or point clouds. Point clouds are the result of advanced surveying techniques that engage laser measuring and photo-stitching, resulting in a 'cloud' of points. This dataset typically has an intermittent existence - its primary purpose is to be translated into polygonal mesh surfaces. This paper outlines pedagogies that ask students to observe and digest the purity of the cloud, developing digital and manual drawing techniques as a means of analysis. Two courses are presented with varying constraints and propose explorative strategies for addressing expansive digital surveys such as point clouds.

How do you observe? When it comes to clouds, an object description involves a self-description.

-Marcel Beyer, Clouds

\section{BACKGROUND - POINT CLOUDS}

A point cloud is a set of data points usually displayed in an XYZ Cartesian coordinate system. Each data point represents a singular moment on the surface of an object, collectively becoming a 'cloud' of information. The generation of point clouds is often associated with survey work where a detailed image of a surface is required. Many disciplines utilize point clouds - archeology, engineering, forensics and medicine to name a few. Typically, the scan and the resulting data is merely the first step - this data is then processed through advanced hardware and software, translating the raw point cloud data to a polygonal mesh. That mesh can be further augmented with texture mapping and other lighting effects to produce a photorealistic model of the scanned object.

While there are multiple scanning technologies, such as photogrammetry, sonar, and CT scanning, laser scanning is one of the most popular methods used to generate point clouds and is extensively utilized in surveying. 3D laser scanning, or LIDAR, is a surveying method that measures the distance to a target by illuminating the target with pulsed laser light and measuring the reflected pulses with a sensor. The varied return times and wavelengths for the laser determine the measured surface's final distance and can be used to make digital 3D representations. The resulting space and colorbased datasets are called point clouds.

There is a beauty to the raw point cloud data. While the point dataset is not the traditional final output, its allure is contagious, and a recently published book, Art of the Point Cloud, celebrates a collection of striking 3D scan graphics. ${ }^{2}$ The laser's simple task, to assess the space and color of one point on a surface, dissolves architectural elements - such as wall, ceiling, and window - blending in equal hierarchy. The field of vision shifts from solid to transparent and back again, allowing viewers to not only perceive the 'real' space but also what lies beyond its solid components.

\section{BACKGROUND - SURVEYING}

Surveying physical space is one of the many practices that has been transformed by this technology. Surveying is the science of determining the three-dimensional positions of points and the distances and angles between them. Land surveying usually occurs surface of the Earth, and they are often used to establish maps and boundaries for ownership, locations, such as building corners or the surface location of subsurface features, or other government and civil requirements. This practice dates back to ancient Egypt, where rope stretchers used knotted cords to measure property demarcations. Drawings of this practice appear in the tombs of scribe Djerserkereseneb and are dated 1400-1390 B.C. Current techniques employ more advanced hardware such as theodolites, a precision optical instrument for measuring angles between designated visible points in the horizontal and vertical planes. Sophisticated total station theodolites include electronic distance measurement (EDM) in their systems, measuring both vertical and horizontal angles and the slope distance from the instrument to a particular point. An onboard computer then collects this data and performs triangulation calculations. ${ }^{3}$

Other survey professions, such as building inspectors, examine architectural objects. The process of building inspection, whether it is by an architect for existing conditions or another position, presents opportunities for misinterpretation of reality. For example, a building fabric inspection has been shown to be to a consistent variable, failing to meet an initial objective. ${ }^{4}$ Straub highlights this situation stating "the practice of condition assessment by building inspectors yields variable results due to subjective perceptions of inspectors. Surveyor variability is defined as the situation where two or more surveyors, 


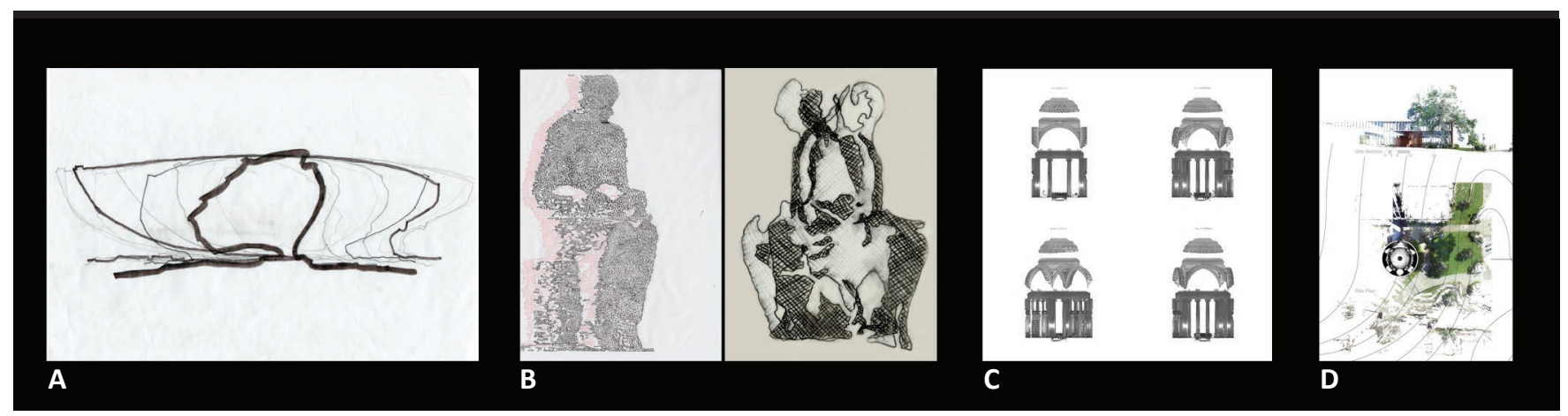

Figure 1. These drawings demonstrate the four assignments from the course: to draw a boat, a set of sculptures, an interior space, and a building.

surveying the same building, arrive at very different survey decisions. This variability is caused by a variety of factors such as previous experience, attitude to risk and, heuristics - the use of 'rules of thumb' - a leaning towards a particular opinion regardless of the available evidence." ${ }^{\prime \prime}$ The scan creates an irrefutable snap of history - a data set that is unbiased and available for multiple interpretations. While each scan requires a person with the same capability of subjectiveness to interpret its data, that initial distillation can be reviewed again, with the help of complex software or other technicians.

Surveying via point clouds has pitfalls, however. This method of documentation captures large amounts of 'useless' data that then requires heavy editing, a problem that is rectified in situ when manually surveying. The processing of $3 \mathrm{D}$ scans requires trained technicians to delete, interpret, and manipulate stray and alien points to parse together a reality. It is in this strange interpretation, the capturing of massive, and potentially unusable, information and then modifying its contents based on one's memory of its reality, that drove its inclusion in a series of architectural studio investigations.

\section{PEDAGOGY OVERVIEW}

The following examples represent two separate approaches in two studios. Each studio engaged a FARO 3D scanner capable of capturing a 50-meter radius of color and volumetric information, producing a cloud of data points that form a targeted assimilation of an object or space. Students are asked to question the truthfulness of the resulting data: like a cloud, its exactness is in a constant state of uncertainty. What is more accurate, the human eye or the projected point? Both are acknowledged as valid.

\section{HANDS ON/HANDS OFF - A 1ST YEAR STUDIO}

In 2016, a first-year summer graduate studio that had emphasized traditional methods of representation was recast. Students were asked to explore a hybrid approach that provides a framework for building digital and analog skills, an understanding of how these modes frame understanding and engagement, and a platform to investigate new ways of representing, experiencing, and designing.
To do this, a digital point cloud scanner was used to record four objects: a boat (figure 1A), a campus sculpture (figure 1B), an interior space (figure 1C), and a small chapel (figure 1D). For each of the three investigations, the students were asked to use digital and manual techniques (point cloud scanner, photo stitching, surveying; hand measuring, sketching, narrative) to document and describe. Each student was required to produce digital (AutoCaAD/Illustrator or similar) and manual (hand drawn) records of the objects that were assessed for clarity, verisimilitude, and accuracy. For each object, students proposed an intervention appropriate to its scale, material, and content. They then presented these in hybrid formats that combined equal effort in digitally produced (renderings, video, 3-D printing, etc) and hand-drawn or modeled work.

In parallel, students investigated Beaux Arts techniques of hierarchy, style, and emphasis, which served as a mechanism for mentally processing the excess data associated with the point clouds. With an emphasis on phenomenology, students used data as a step towards affect.

The semester's last half was dedicated to the final project, designing a building. This intervention asked the students to understand interior and exterior conditions of Eero Saarinen's Oreon Scott Chapel located on Drake University campus in Des Moines. Starting with an introduction to Beaux-Arts principles and techniques, students scanned and sketched the chapel's interior and the exterior in order to inform design and representation. This project eliminated dimensional limitations. Proposals could be as small or large as the student deemed appropriate. Each student presented hand-drawn perspectives, Beaux Arts case studies, architectural case studies, one digital/analog physical model that expanded on earlier procedural technique, a digital model exported to Google cardboard that provided a virtual immersion in the revised space, and three 'digital beaux-arts' drawings; black and white drawings with no fewer than five line weights plus shading and texture as appropriate; in plan and section demonstrating the intervention's configuration. 


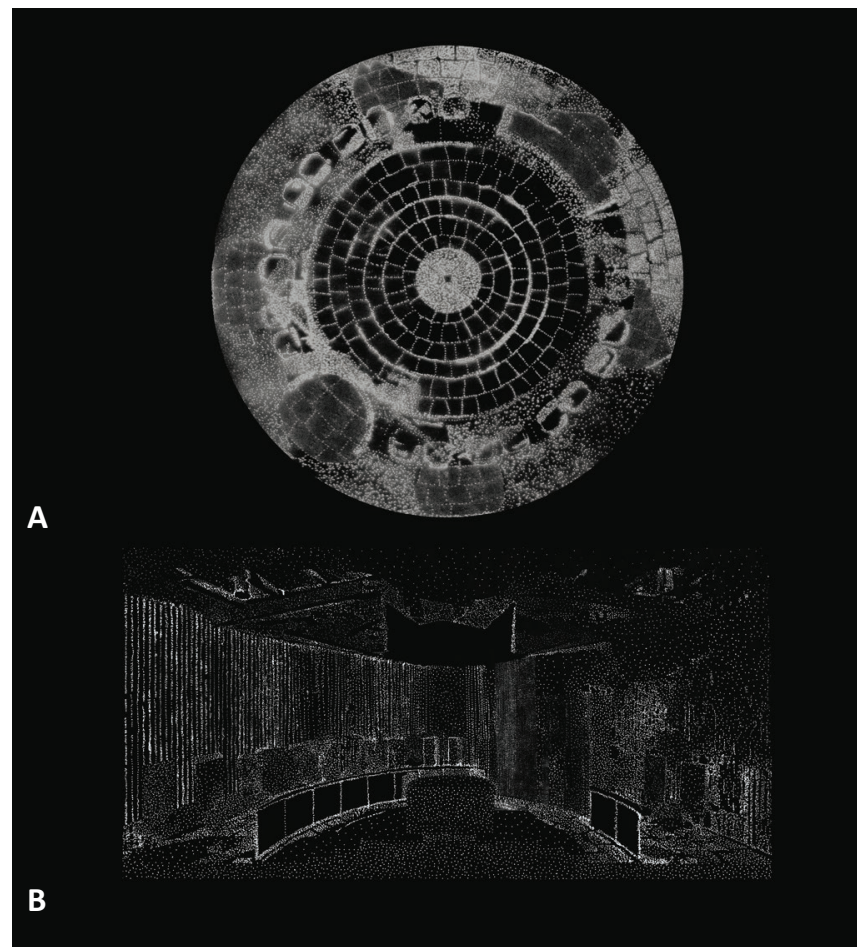

Figure 2. A final project from the studio investigating the space of stippling

Erin Hunt's project focused on the interrogation of a manual technique against observations of the point cloud and allowed it to inform her design proposal. Point clouds can provide a full understanding of space with massive information collected from individual points. Even if there is an absence of information, which often occurs with this documentation methodology, the eye can fill the gap. Erin proposed an intervention that similarly provided gaps that were visually understood as a whole. During the first day drawing workshop, Erin embarked on a representation through stippling, a drawing method that uses small dots to simulate varying degrees of solidity or shading, drawing direct correlations between the points of the point cloud and the point of the stipple (figure 2A). Her first investigations were not focused on any specific design intent but the retranslation of the given point cloud through hand rendering techniques. Focusing on points of views informed by cinematic methods and cubism, she chose the perspective as her way into the design strategy (figure 2B). Point clouds are notorious for their information overload; they collect data indiscriminately, such as out a window or off a mirror, and this data is often perceived as excess. Erin used this manual transcript as an opportunity to edit and fully engage the existing conditions. She engaged a range of tracing strategies, both directly from a laptop screen with trace paper and pen, as well as projection on a vertical surface. Both presented opportunities to make calculated edits, with each recorded stipple an act of remembering and each dismissed point an act of omittance. As her project progressed, she considered the concept of fractures and materially cuts into the building by creating apertures, noting that the symmetry of the chapel would allow for re-creating the 'missing pieces'. However, her project's final manifestation was an edited immersive and experiential chapel, as the translation from the point cloud's survey and the stippling was the design process at hand. At this intersection, questions emerged: would this immersive experience be physical or digital? What will the next translation be? Her project resonated with an idea of distilling moments out of universal principles and this narrative proved for a particularly engaging presentation.

Hands-On/Hands-Off explores the limits of digital and manual languages and critically assesses how drawing can initiate dialogue about human productions that exist between the intellectual and the sensory. Parallels between the two-as well as contradictions-form the conceptual heart of the work, providing students with a critical but not skeptical basis for future investigation.

\section{ARCHITECTURE X LANDSCAPE - A 3RD YEAR STUDIO}

In the undergraduate program, Arch 301 represents an important transition from the introductory and fundamental emphases of the 1 st and 2 nd year studios. Students focus on the intersection of architecture and landscape as constructed, cultural artifacts, developing a personal philosophy of architecture that reflects their values and proclivities.

In the Fall of 2017, a group of students moved into the scanned landscape and were asked to analyze through creative action as a means of reinterpreting the proposed site. Working in pairs, the students operated on, distorted, dissected, expanded and systematically mutated through modeling, drawing, and projection. This project initiated with an introduction to point cloud manipulation through programs such as FARO and Cyclone, and manual techniques through projection mapping and drawing. Each pair of students sought out a specific microsite in Ledges State Park, a large expansive national park in lowa. The park contains a sandstone gorge carved by Pea's Creek, a tributary of the Des Moines River. The gorge is up to 100 feet deep with concretions jutting from the cliffs, an atypical land formation for lowa. After specific sites were identified and scanned, the students were asked to create large 3' $x 6^{\prime}$ hand drawings developed through point cloud manipulation and projection and smaller digital drawings and diagrams.

First, students were asked to use these point cloud datasets as the basis of diagramming. This was an open opportunity to explore the aesthetic, texture, and form of point cloud geometry as generative diagrams for design catalyst. Rather than manual editing, students were encouraged to edit unwanted point noise in the provided point cloud software - by exploring the cloud, students delete points until they have a cohesive objects and surfaces. This was then output as meshes to other software for visual and representational exploration. Gregory Schuab's project investigates light and 


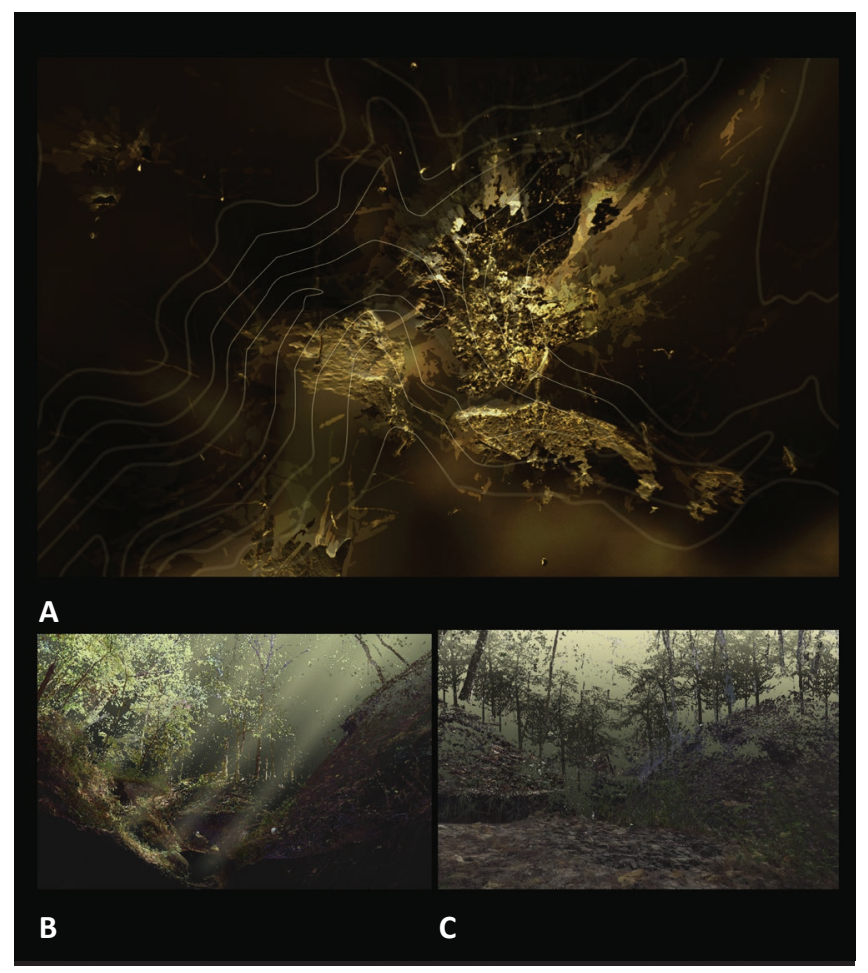

Figure 3. Renderings of tree canopies generated from point cloud scans.

shadow of his point cloud set: by generating surfaces from overhead canopies, he assigns materials and lighting effect via VRay in Rhinoceros, producing a site plan dedicated to the shade of the September foliage (figure 3A). Greg's subsequent drawings articulated in perspective the dappling of light produced under a tree canopy (figure 3B). These investigations served as a basis of lighting conditions for his design strategies. Alec Hoppe chose to investigate texture at different edge conditions on the site. These studies provided material understanding, informing the texture of his design intervention (figure 4A). Kylee Cangas used her point clouds as a canvas for diagramming, integrating design elements, water flow data, and other information layered over the point cloud (figure 4B).

The large manually-edited drawings required the students to choreograph a model manipulation of the point clouds, such as a sequence of perspectival views, roundtable animations, or serial section cuts, and use these frames as a background for manual exploration. Using unedited point clouds, students captured a series of images that provided opportunities for investigation. Students were immersed in the point cloud system via projection of the captured images, inhabiting the model beyond the limitations of a flat screen. Working in pairs, students developed drawing methods for individual characteristics to reinforce a thematic topic in their research. Different engagements emerged by representing and recording data in a variety of ways such as watercoloring, hatching, stippling and outlining. For example, Kylee Cangas and Quinn Lyon's outlined serial sections in both plan and section, resulting in

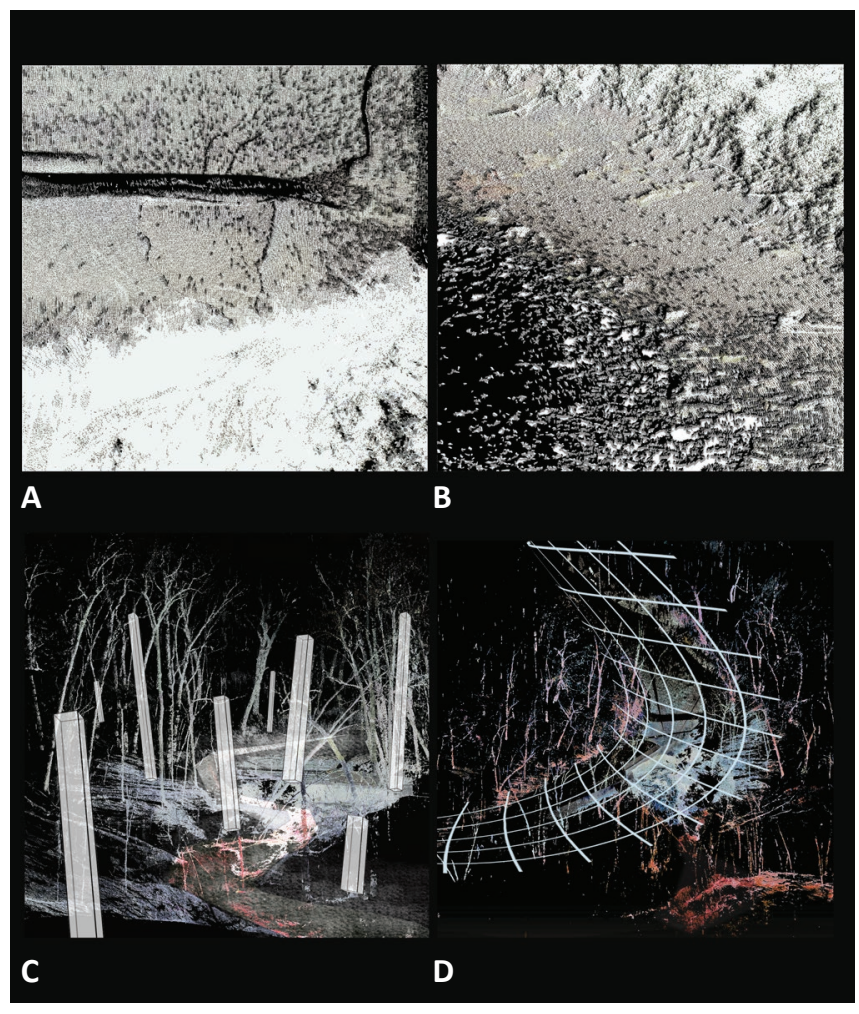

Figure 4. Drawings demonstrating different diagramming styles.

a flattened investigation of section and greenery (figure 5A). This was distilled through ink and watercolor, denoting the fluctuating pattern of section through the ground as well as the vegetation. Michael Oddo and Gregory Schuab continued to investigate the canopy, generating a filigree of leaf outlines that dapple the leafy overhead (figure 5B). All of these drawings vary in context but seek to find a new way of engaging the abundant data that accompanies LIDAR scans.

\section{CONCLUSION}

These of drawings initiate in a state of lostness, which in turn provides a space that is unrestricted of assumptions. By combining manual and digital principles, these studios navigated a careful balance between tradition and experimentation, offering students a palette of tools and mindsets that resonate in contemporary education and practice. While enthusiasm for digital techniques is well-warranted, their implementation in studio courses in particular often squeezes out discussion of other methods and value systems. The proposed processes embrace progress of technology while engaging a traditional method of distillation that is often referred to as lost. These two studios open up a dialogue with technology, rather than because of it or in use of it. These sites, in their digitized nebulous forms, provided a varied perspective, unconsumable and requiring dissection. In the words of Marcel Beyer, "If you devote yourself to clouds and their study, you're lost." These projects celebrate lostness and their subsequent journeys, providing workflows for a deeper comprehension of a space or object, resulting in design catalyst. 


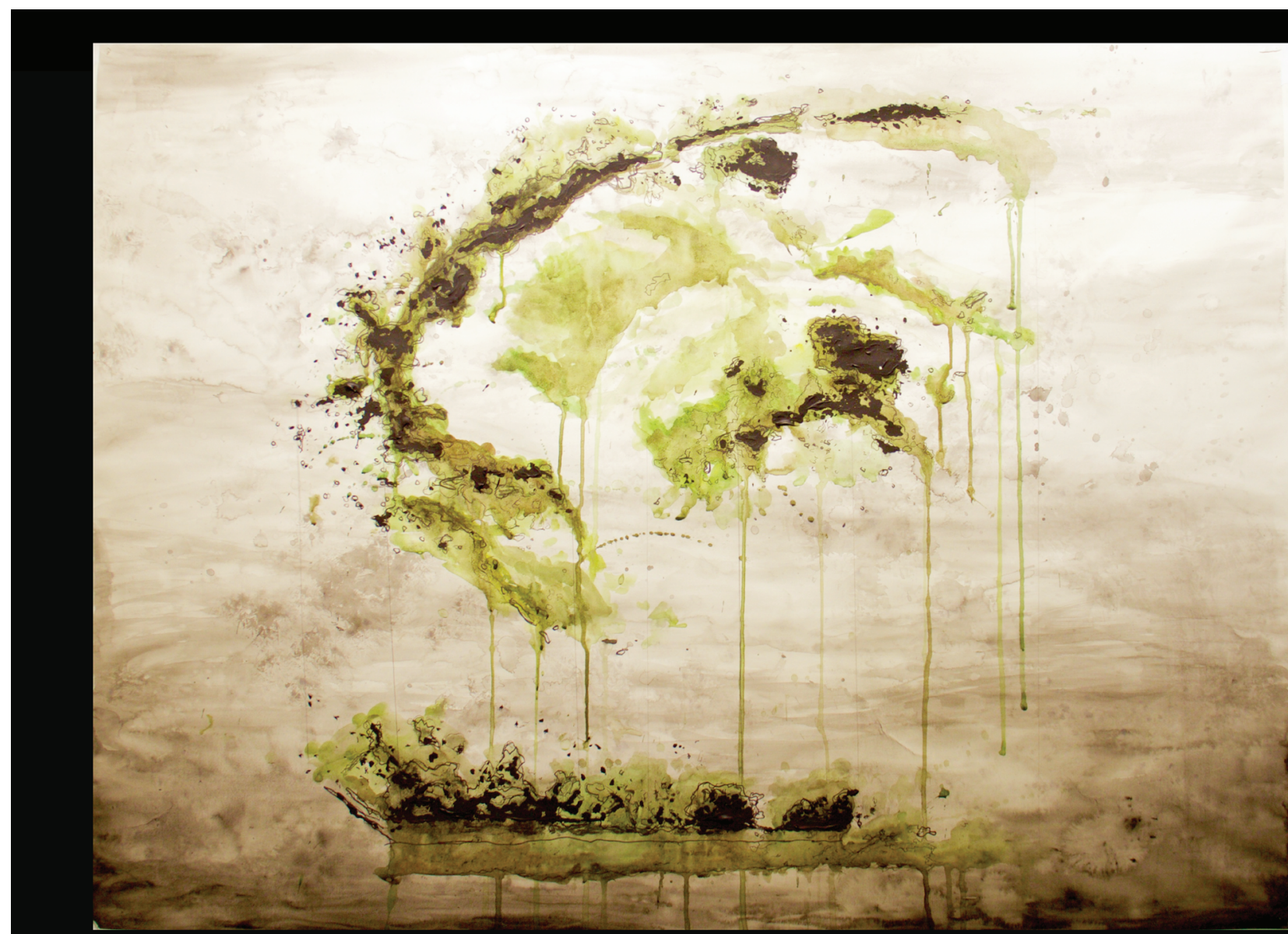

A

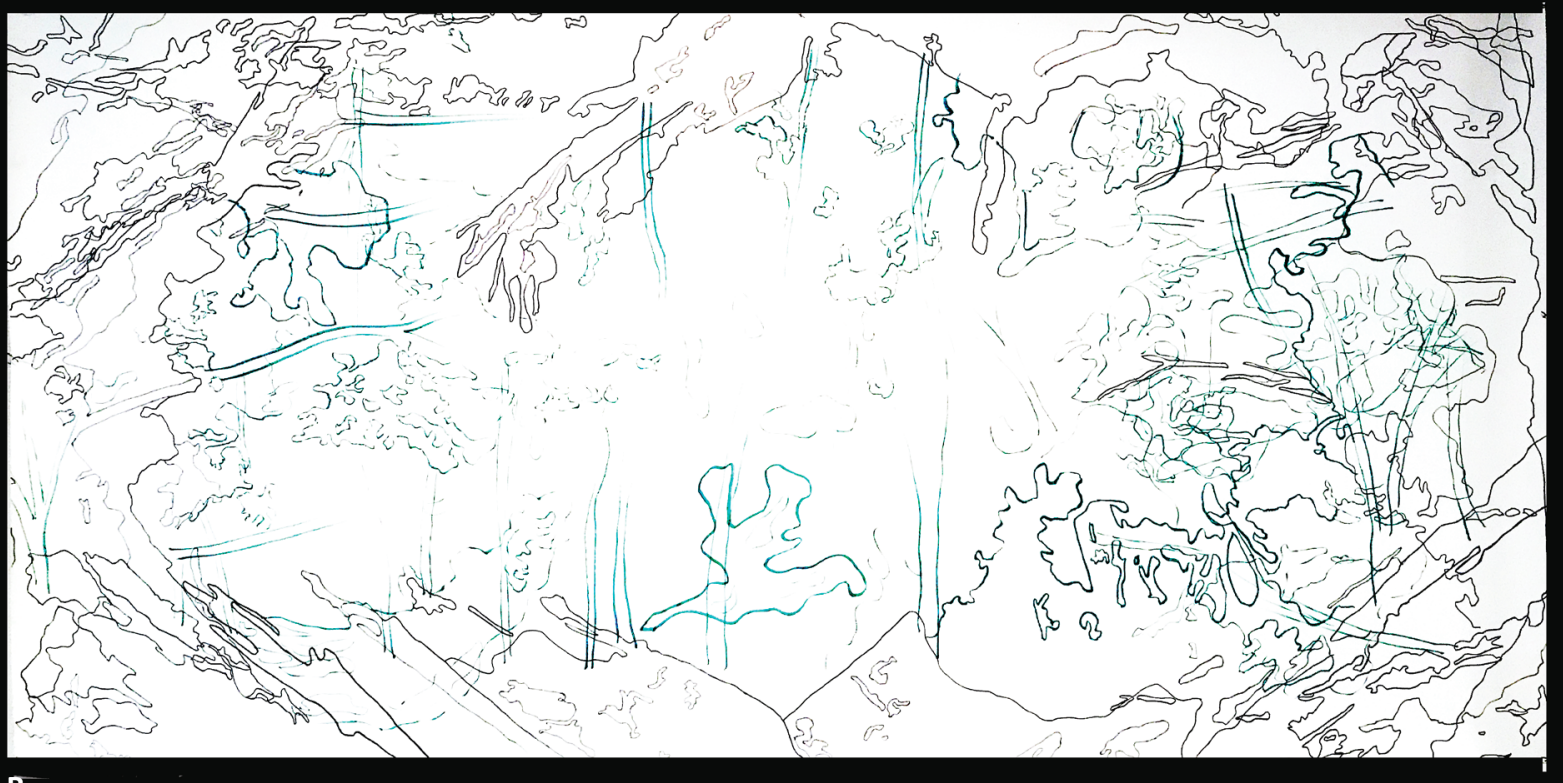




\section{ENDNOTES}

1 Marcel Beyer and Helmut Völters. Wolkenstudien = Cloud Studies = Etudes des nuages (Germany: Spector Books, 2013).

2 Paul Chapman, et al., Art of the Point Cloud: A Celebration of Some of the Most Beautiful and Striking 3D Scans from around the World (Wild Harbour Books, 2018).

3 Barry F. Kavanagh and S. J. Glenn Bird, Surveying: Principles and Applications, fourth edition (Upper Saddle River, NJ: Prentice Hall, 1996).

4 Bosché, Frédéric, Alan Forster, and Enrique Valero, 3D Surveying Technologies and Applications: Point Clouds and Beyond, Technical paper, Royal Academy of Engineering Centre of Excellence in Sustainable Building Design, CyberBuild Research Lab, Heriot-Watt University, 5-7.

5 Ad Straub, "Dutch Standard for Condition Assessment of Buildings," Structural Survey 27, no. 1 (2009): 23-35. 